\title{
FORCE FREE DESIGN OF SUPER-FERRIC BEAM LINE MAGNET
}

\author{
M. Wake, H. Piekarz , R. Yamada ${ }^{*}$, H. Sato and K. Takayama \\ KEK, Tsukuba, 305-0801 Japan, FNAL ${ }^{*}$, Batavia, IL 60510, USA
}

\begin{abstract}
A force free design of super-ferric beam line magnet is proposed. Super-ferric magnets use induction efficiently to produce magnetic field. The problem of super-ferric magnet has been the electromagnetic force that increases the heat load of the magnet and eliminates the advantage of the energy saving of superconductivity. This design balances out the electromagnetic force works on the conductor and realizes the very light support of the conductor resulting a very small cold mass and heat load. Beam line magnet can be much more economical both in construction and operation. Shifting of conductor position, asymmetric pole width and adjusting holes in the pole piece are the major force balancing techniques. Testing is planned using VLHC test facility at Fermilab
\end{abstract}

\section{INTRODUCTION}

Beam line magnet has been an attractive application area of superconducting magnet. High ramp rate that generate transient loss of superconductor is not required in most of the beam lines. Power saving of superconducting magnets in DC operation is very large. However, construction cost of superconducting magnets and operation cost of cryogenic system has been expensive enough to hesitate the use of superconducting magnets unless the requirement of the bending field is very high.

One of the solutions to use suprconducting magnet efficiently at low field is the use of iron yoke. Super-ferric magnet should be very economical because it uses much less superconductor. The problem of superferric magnet is the cryogenics. Cryostat containing whole iron is costly and lossy. Warm iron design has a problem of large electro-magnetic force between iron and conductors. Heavy-duty spacers to support coils in a vacuum insulation easily give a very large heat load to the cryogenic system. Cost down merit of super-ferric magnet is difficult to achieve in both case.

The design presented in this paper balances out the electro-magnetic field by two gaps of iron. Since support system is very simple and light, cryogenic load is small and construction cost is very low. The principle of the force balance is similar to the VLHC Pipetron magnet but the construction of the magnet is made asymmetric so that it becomes suitable for single bore beam line magnets.

\section{DESIGN PRINCIPLE}

The electromagnetic force on the magnet conductor is the vector product of field and current. For example, 20 turns of $5 \mathrm{kA}$ in $2 \mathrm{~T}$ field results in $200,000 \mathrm{~N}$. It easily becomes very large. This is the reason why we need to design magnets with very rigid supporting structure to prevent quenches due to conductor motions. However, if the direction of the field is opposite at both sides of a conductor, the total force working on the conductor could be balanced out. This is automatically achieved in a symmetric structure having a single conductor in the center of the collider geometry. The pipetron magnet, or transmission line magnet, in the VLHC phase I design, is based on this principle [1]. Although it requires 100kA class high current, $2 \mathrm{~T}$ magnetic field in a $2 \mathrm{~cm}$ iron gap was successfully achieved by a single conductor. Support structure of the conductor of this magnet was very simple and light with small heat load.

Two-aperture geometry of pipetron magnet requires twice large current to generate field. Therefore, it is not ideal to use such two-aperture magnet for a beam line, which uses only one aperture. Because most of beam lines require larger pole gap, typically $40 \mathrm{~mm}$, operation current will become too large to operate. Although we have solution for it, high current is the major disadvantage of this design. Since the electro-magnetic force on the conductor is generated by the leakage field comes from the pole gap, one gap which is not used for beam can be smaller than other gap if the conductor position is shifted closer to the smaller gap. The basic geometry of this magnet is shown in Fig.1. Conductor is the only cryogenic part of the magnet. It is supported in a vacuum jacket covered by super-insulations and cooled by the liquid helium flow in the conductor. Other parts all stay at room temperature. Therefore, the cold mass of the magnet is very small.

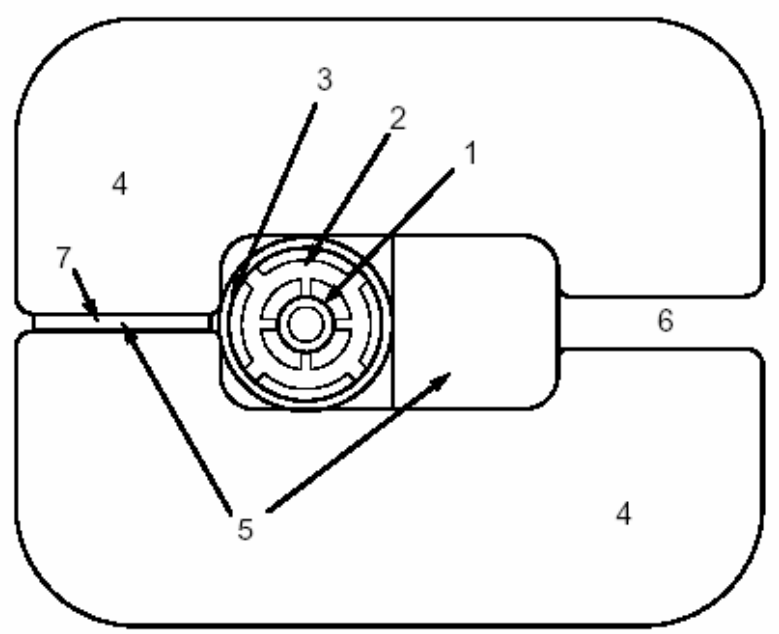

Fig. 1: Asymmetric force balance structure.

1:Superonducting cable 2:Conductor support 3:Vacuum tube for thermal insulation 4:Iron yoke 5:Iron support structures made of non-magnetic stainless steel. 6:Beam gap 7:Dummy gap to generate counter force 
The conductor is made of superconducting braid sandwiched between two invar pipes so that the thermal contraction is avoided. The beam tube is placed in the large gap. The small gap works as the dummy gap to generate the force balance field.

\section{FORCE BALANCING}

The problem of the asymmetric construction is the saturation of iron. In a symmetric geometry, force balance can be maintained no matter how iron is saturated but the force balance can not be automatically maintained in asymmetric geometry when both side of iron saturate differently. The calculation of magnetic field and the force on the conductor has to be made using finite element code with non-linear iron permeability. We used ANSYS 6.2. The optimum design should be determined by looking at the force as a function of the current.

Figure 2 is the plot of the force on the conductor as a function of the central field of the beam space. Curves are plotted for different dummy gaps. Apparently, the force balance changes with the current. Forces are not completely eliminated. However, the level of the force is in the order of several hundred Newton's if the gap is adjusted to the optimum for the field of up to 1.5 Tesla. This is not large and practically acceptable for a light spacer system. Since conventional magnets are excited often to the saturation level, it is desired to go to higher field to compete with conventional magnets in the construction of beam lines.

There is a way to control the saturation of iron to some extent. The force calculation result after saturation control is shown in Fig.3. If the width of pole piece is wide, the saturation starts at higher current. If a hole is made at the edge of the pole piece, local saturation of iron provide counter electro-magnetic force at lower level of excitation.

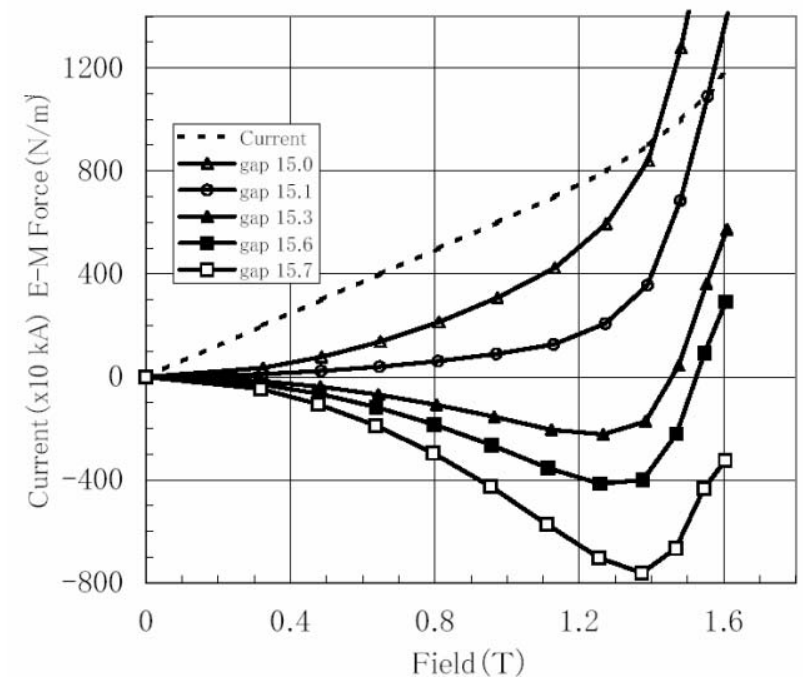

Fig. 2: Electro-magnetic force in asymmetric construction

Forces for different dummy gaps are plotted. Half gap $15.3 \mathrm{~mm}$ provides optimum force balance up to $1.5 \mathrm{~T}$ with maximum force $-200 \mathrm{~N}$.
Combining such techniques, one can design a no-force magnet for a single aperture beam line magnet. Figure 4 is the cross section of the design example. Flux line pattern in the optimised geometry is also shown in the figure. It is shown that the force balance is well maintained even after a severe saturation of iron started at $1.5 \mathrm{~T}$. Essentially there is no force up to 1.7T. The vertical force is zero if the conductor is in the vertical center but this is an unstable balance. De-centering force of $2 \mathrm{kN} / \mathrm{mm}$ works on the conductor. Therefore, vertical alignment of less than $0.1 \mathrm{~mm}$ is required to stay within $200 \mathrm{~N}$, although the field quality is not much affected by the position of the conductor. The conductor support can achieve this accuracy [1]. The beam gap of the magnet in Fig.4 is $140 \mathrm{~mm} \times 40 \mathrm{~mm}$. Operation current is $129.2 \mathrm{kA}$ at $1.7 \mathrm{~T}$. Most of beam line requirement is satisfied by this design. For example, a beam line of $50 \mathrm{GeV}$ proton with bending radius $110 \mathrm{~m}$ needs $1.514 \mathrm{~T}$ in the average. If magnetic field of $1.7 \mathrm{~T}$ is provided in the magnet, $20 \mathrm{~cm}$ spaces are available between every magnet of length $2 \mathrm{~m}$. Monitors and other instrumentation can be installed in these spaces. One side of beam tube is opened and accessible in this magnet and the necessary spaces in between magnets are not as much as $\mathrm{H}$ shape magnets. Since the field of this magnet is generated by single-conductor cable, quadrupole magnet of this type is not possible. Alternate gradient configuration is necessary to make magnet combined with the focusing capability.

\section{OTHER ADVANTAGES}

By moving the conductor from the center to the asymmetric position, the space for the structure to connect top and bottom iron became available.

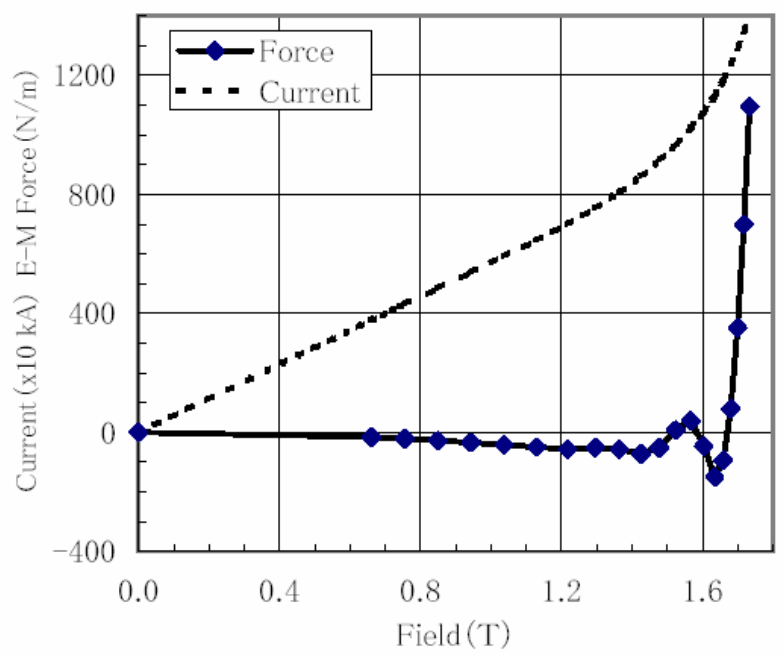

Fig. 3: Electro-magnetic force in adjusted construction

Pole width $200 \mathrm{~mm}$ and half gap $13.2 \mathrm{~mm}$ with $5 \mathrm{~mm}$ diameter hole in the dummy pole piece gave optimum. 
With the combination with the spacer in the dummy gap, both iron yokes can be firmly connected. Since the field quality of this magnet is dependent on the iron shape, and the force between two irons is very large, such structure is helpful to achieve the field quality.

It is important to reduce unnecessary field in produced by the current at the spaces in between magnets. If we use symmetric two gap magnet, it is difficult to shield the beam tube from the field coming from the conductor. Beam tube is too close to the conductor. In this design, the distance between the beam tube and the current provide the space for shielding. Even just rotating the iron structure by 90 degree would provide a nice magnetic shield with no-force on the conductor.

The connecting structure of iron also works as the radiation shield. Since conductors are exposed to the beam space in usual magnet design, beam induced quenches are serious problems for superconducting magnets. Magnets are often designed with extra large aperture to avoid beam induced quenches and this causes high cost of production and operation. But this magnet hide superconductor behind the thick stainless steel block and there is no problem of beam induced quenches.

\section{OPERATIONAL}

To operate this magnet in a beam line, we need to set up the vacuum insulated conductor along the beam line just like a power transmission line. Iron yokes covering this transmission line will simply make the magnet strings. The return current bus has to be made separately. There is a possibility to use return current but the contribution of the return current to the single aperture magnet is very little [2].

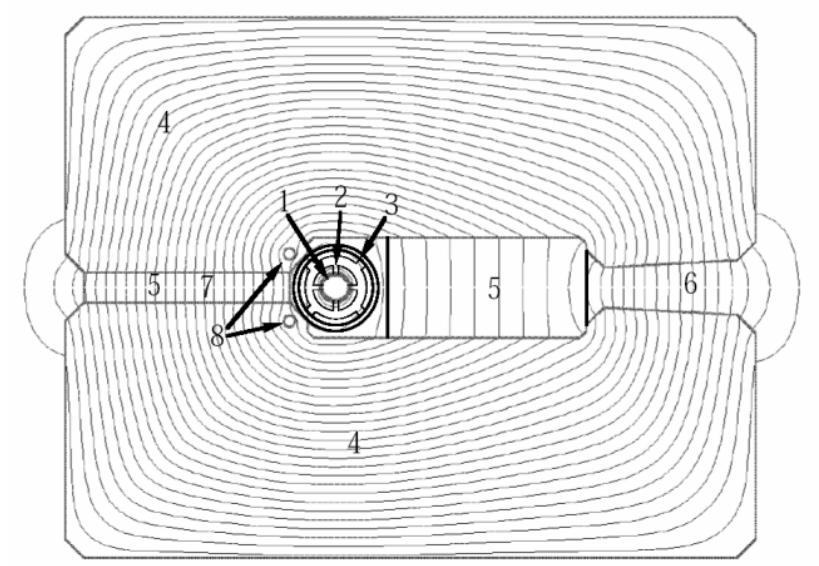

Fig. 4: No-force cross-section.

1:Superonducting cable 2:Conductor support 3:Vacuum tube for thermal insulation 4:Iron yoke 5:Iron support structures made of non-magnetic stainless steel. 6:Beam gap 7:Dummy gap to generate counter force 8:Holes in the dummy pole piece to control the saturation of iron
Very simple structure of the magnet makes the construction cost very reasonable. However, the operation of this magnet requires a very large current. At low field, $100 \mathrm{kA}$ is not a very large current for superconductor. The current carrying capacity of superconductor increases very much at low field. A test of conductor operation for pipetron was successfully made including joints at Fermilab [1]. In case of very long beam line, Nb3Sn can be used to run the system with elevated temperature [3].

Thermal contraction in thick straight line is difficult to handle but the transmission line conductor uses invar pipes both for liquid helium channel and the pressure pipe so the contraction effect is minimized to the level that mechanical stresses resulting from contraction/expansion can be well controlled. A 100kA power supply is technically not difficult but costly if it is done in conventional way. We have introduced a special power supply system. Even the current is high, the voltage required to excite this magnet is very low. Recent development of IGBT chopper and shotky diode enables us to build low voltage high current power supply. A 100kA power supply was already built at Fermilab. KEK demonstrated feasibility of such a power supply using a smaller version [4]. Current leads to supply $100 \mathrm{kA}$ into the conductor have been fabricated at Fermilab and will be tested soon. The cryogenic heat load of the current lead is about $1 \mathrm{kw}$ including the helium flow in the leads and this is the main part of the heat load. Still total heat load is not as much as the usual superconducting magnet if the length of the beam line is sufficiently long.

\section{CONCLUSION}

The force free design of superferric beam line magnet was successfully performed. A test operation of this magnet is being prepared using the VLHC test bench at Fermilab. Detailed force balance characteristics will be studied in the test operation. If this design is applied in some of the accelerator beam line, it becomes a good performance proof of the VLHC phase I superconducting magnet system for the operation economy and long term reliability.

\section{REFERENCES}

[1] "Design Study for a Staged Very Large Hadron Collider", Fermilab-TM-2149, 2001.

[2] M.Wake and R.Yamada, "New Types of Superconducting Magnet Designs for High Energy Collider", Proc. MT-15 p.47-50, Science Press, Beijin, 1998.

[3] E.Barzi, G.W.Foster, E.Malamud, P.O.Mazur, H.Piekarz, M.Wake, K.Hayashi, M.Koganeya, "Nb3Al Prototype Conductor for the Transmission Line Magnet", proc.1999 Particle Accelerator Conf. 3330-3332,1999.

[4] M.Wake, H.Sato, K.Suda, "A Battery-Powered HighCurrent Power Supply for superconductors (in Japanese)" Teion-kogaku, 37 783-786,2002. 